\title{
Reverse quenching in a one-dimensional Kitaev model
}

\author{
Uma Divakaran, 1 a and Amit Dutta ${ }^{1,0}$ \\ ${ }^{1}$ Department of Physics, Indian Institute of Technology, Kanpur 208 016, India
}

(Dated: October 11, 2018)

\begin{abstract}
We present an exact result for the non-adiabatic transition probability and hence the defect density in the final state of a one-dimensional Kitaev model following a slow quench of the parameter $J_{-}$, which estimates the anisotropy between the interactions, as $J_{-}(t) \sim-|t / \tau|$. Here, time $t$ goes from $-\infty$ to $+\infty$ and $\tau$ defines the rate of change of the Hamiltonian. In other words, the spin chain initially prepared in its ground state is driven by changing $J_{-}$linearly in time up to the quantum critical point, which in the model considered here occurs at at $t=0$, reversed and then gradually decreased to its initial value at the same rate. We have thoroughly compared the reverse quenching with its counterpart forward quenching i.e., $J_{-} \sim t / \tau$. Our exact calculation shows that the probability of excitations is zero for the wave vector at which the instantaneous energy gap is zero at the critical point $J_{-}=0$ as opposed to the maximum value of unity in the forward quenching. It is also shown that the defect density in the final state following a reverse quenching, we propose here, is nearly half of the defects generated in the forward quenching. We argue that the defects produced when the system reaches the quantum critical point gets redistributed in the wave vector space at the final time in case of reverse quenching whereas it keeps on increasing till the final time in the forward quenching. We study the entropy density and also the time evolution of the diagonal entropy density in the case of the reverse quenching and compare it with the forward case.
\end{abstract}

PACS numbers: 73.43.Nq, 05.70.Jk, 64.60.Ht, 75.10.Jm

\section{INTRODUCTION}

Studies of quantum phase transitions in quantum many particle systems have always been a fascinating area of research in condensed matter physics [1, 2]. While a plethora of theoretical works have been performed on statics of quantum phase transitions (QPT), the dynamics of a quantum system passing through a quantum critical point has caught the attention of researchers only recently 3, 4, 5, 6, 7, 8, 9, 10, 11, 12, 13, 14, 15, 16, 17, 18, 19, 20, 21, 22, 23, 24, 25, 26, 27]. Understanding quantum dynamics happens to be a challenging problem as the physics of equilibrium quantum phase transitions gets coupled to the non-equilibrium dynamics of correlated systems. Theoretical techniques used in studying the above dynamics are often borrowed from similar studies in quantum optics, e.g., the Landau-Zener transition [28, 29].

The dynamical evolution can be initiated in a quantum system either by a sudden change of a parameter in the Hamiltonian which is called a sudden quench [4], or by a slow quenching of a parameter [5, 6]. The effect of the passage through a quantum critical point is manifested in the eventual dynamics of the system. The relaxation time $\left(\xi_{t}\right)$ of the system, defined as the time taken by the system to come back to its equilibrium state after a small perturbation, diverges at the critical point as $\xi_{t} \sim \delta^{-\nu z}$ where $\delta$ measures the deviation from the quantum critical point and $\nu$ and $z$ are the correspond-

\footnotetext{
*Electronic address: udiva@iitk.ac.in
}

${ }^{\dagger}$ Electronic address: dutta@iitk.ac.in ing correlation length and dynamical critical exponents, respectively. The divergence of the relaxation time is an artifact of vanishing energy gap $\Delta\left(\sim \xi_{t}^{-1}\right)$ between the ground and the first excited state of the Hamiltonian near a quantum critical point. This divergence of the relaxation time forces the system to be infinitely sluggish near the critical point so that it takes effectively an infinite time to respond to any change in the external parameters thereby causing excitations. The recent discovery of ultra cold atoms which has facilitated the experimental implementation of various Hamiltonian models [30, 31] and thus the verification of results of quantum dynamics, has enormously accelerated the theoretical research in this field. Here, we are interested in a slow and linear variation of the quenching parameter and estimate various quantities such as density of defects and the local entropy density in the final state of the system following a quench, as a function of the quenching rate $\tau$.

It is well known that for a $d$-dimensional system which is initially prepared in its ground state and is quenched through a quantum critical point by linearly varying a parameter as $t / \tau$, the density of defects $(n)$ satisfies the Kibble-Zurek(KZ) scaling [5, 6, 15, 32, 33] given by $n \sim \tau^{-d \nu /(\nu z+1)}$ where $\nu$ and $z$ are the critical exponents defined above. The Kibble-Zurek scaling has been verified in various exactly solvable spin models [5, 7, 9, 12, 13] and in a system of interacting bosons undergoing superfluid to insulator transitions [6]. The above KZ scaling relations gets modified when the system is quenched through a multicritical point 25], across a gapless phase [16, 18], along a gapless line [21, 23] or for quenching with a non-linear rate [17]. Studies on quenching dynamics have also been generalized to quantum spin chains with quenched disorder [10], to systems in presence of white 
noise 14], to systems with infinite range interactions [22], to an open system coupled to a heat bath [19], and also to quantum spin chains driven by an oscillatory magnetic field [26] . The effect of edge states on the defect production has also been studied [27]. It is worth mentioning here that the defect production has been studied experimentally for a rapid quench in a spin- 1 bose condensate 34.

In this paper, we study the effect of the reversal of the quenching path right at the quantum critical point on the density of defects. This is achieved by increasing the quenching parameter from time $-\infty$ to its value at the quantum critical point and the bringing it back at the same rate to its initial value at the final time i.e., $t \rightarrow \infty$. We call this quenching scheme as reverse quenching whereas the other scheme in which the quenching parameter is monotonically increased from time $-\infty$ to $+\infty$ through a quantum critical point will be referred to as the forward quenching scheme. We would also occasionally use the term half quenching for the case when the quenching is stopped at the quantum critical point.

At the outset, let us discuss the motivation behind our study. The forward quenching scheme has been applied extensively for the entire range in time from $t=-\infty$ to $\infty$ and defects generated in the final state has been estimated. In the present work, we drive the system linearly right up to the quantum critical point and then let it retrace its path. In our calculation, we find that the defect generated up to time $t=0$ is approximately half of the defect generated for a full forward quenching. We also seek answer to the question that how the defects generated in the first half of the quenching get altered under reversal of the path! We address questions like do we have a similar scaling form for the defect or how does the magnitude of the defects in the final state, i.e. at $t \rightarrow \infty$ change under reverse quenching?. In the process, we also provide an exact solution of the Schrödinfer equation to find the non-adiabatic transition probability for the reverse quenching.

We also compare our work with that in ref. [20] where a quantum XY spin chain is repeatedly swept through the quantum critical points by varying the magnetic field at a linear rate between $-\infty$ and $\infty$ such that the reversal of path takes place far away from the critical points. In the present work, the parameter is increased only upto the quantum critical point where it is reversed to trace back its path. To employ the reverse quenching scheme in an appropriate way, we study the dynamics of a onedimensional Kitaev model where the quantum critical point occurs at $t=0$. Secondly, in ref. [20], the defect density after each repetition is estimated using a recursive relation for the non-adiabatic transition probabilities where the (rapidly varying) cross terms are ignored because they vanish upon integration over the wave vectors. On the other hand, we present here an exact solution of the transition probabilities which include interference terms although the results are in fairly good agreement at least qualitatively with the one cycle case discussed in ref [20] It is also to be noted that the reverse quenching dynamics has been studied for a generic two level system in ref. [35] and the excitation probability has been calculated within the framework of perturbation theory. On the other hand, we here generalize the quenching scheme to a many particle system and solve the Schrödinger equations exactly.

The paper is organized in the following way: the model and the quenching scheme are discussed in section II. The main results for the non-adiabatic transition probability, defect density and the entropy density are presented in section III. We summarize our results in the concluding section whereas the calculational details are provided in the appendix.

\section{THE MODEL AND THE QUENCHING SCHEME}

Two-dimensional Kitaev model defined on a honeycomb lattice described by the Hamiltonian [36]

$$
\tilde{H}=\sum_{n+l=\text { even }}\left(\sigma_{n, l}^{x} \sigma_{n+1, l}^{x}+J_{2} \sigma_{n-1, l}^{y} \sigma_{n, l}^{y}+J_{3} \sigma_{n, l}^{z} \sigma_{n, l+1}^{z}\right)(1)
$$

where $n$ and $l$ defines the column and row indices of the lattice has been studied extensively due to its exact solvability by Jordan-Wigner transformation [37]. The rich phase diagram of this model has a gapless phase, through which the quenching dynamics has been studied recently [16]. The one dimensional version of the Kitaev model (with $J_{3}=0$ ) given by the Hamiltonian [36, 38]

$$
H=\sum_{n=1}^{N}\left(J_{1} \sigma_{2 n}^{x} \sigma_{2 n+1}^{x}+J_{2} \sigma_{2 n-1}^{y} \sigma_{2 n}^{y}\right)
$$

where $n$ refers to the site index, exhibits a quantum phase transition at $J_{1}=J_{2}$. The above Hamiltonian (2), which is the model of interest in this work, can be exactly diagonalized by standard Jordan Wigner transformation [37] as defined below

$a_{n}=\left(\prod_{j=-\infty}^{2 n-1} \sigma_{j}^{z}\right) \sigma_{2 n}^{y}, \quad b_{n}=\left(\prod_{j=-\infty}^{2 n} \sigma_{j}^{z}\right) \sigma_{2 n+1}^{x}$.

Here $a_{n}$ and $b_{n}$ are independent Majorana fermions at site $n$ [16]. They satisfy the relations like

$$
\begin{array}{r}
a_{n}^{\dagger}=a_{n}, b_{n}^{\dagger}=b_{n},\left\{a_{m}, a_{n}\right\}=2 \delta_{m, n}, \\
\left\{b_{m}, b_{n}\right\}=2 \delta_{m, n},\left\{a_{m}, b_{n}\right\}=0 .
\end{array}
$$

Substituting for $\sigma_{n}^{x}$ and $\sigma_{n}^{y}$ in terms of Majorana fermions followed by a fourier transformation, Hamiltonian (2) can be written as

$$
H=2 i \sum_{k=0}^{\pi}\left[b_{k}^{\dagger} a_{k}\left(J_{1}+J_{2} e^{i k}\right)+a_{k}^{\dagger} b_{k}\left(-J_{1}-J_{2} e^{-i k}\right)\right]
$$


where the fourier component $a_{k}$, satisfying the standard anticommutation relations $\left\{a_{k}, a_{k^{\prime}}^{\dagger}\right\}=\delta_{k, k^{\prime}}$ and $\left\{a_{k}, a_{k^{\prime}}\right\}=0$, is defined as

$$
\begin{array}{r}
a_{n}=\sqrt{\frac{4}{N}} \sum_{k=0}^{\pi}\left[a_{k} e^{i k n}+a_{k}^{\dagger} e^{-i k n}\right] \\
+\sqrt{\frac{2}{N}}\left[a_{0}+a_{0}^{\dagger}+a_{\pi}(-1)^{n}+a_{\pi}^{\dagger}(-1)^{n}\right] .
\end{array}
$$

The sum over $k$ in Eq. (6) goes only for half the Brillouin zone as $a_{n}^{\prime} s$ are Majorana fermions. By defining $\psi_{k}=$ $\left(a_{k}, b_{k}\right)$, the Hamiltonian (5) can then be rewritten in a simpler form as

$H=\sum_{k=0}^{\pi} \psi_{k}^{\dagger} H_{k} \psi_{k}$

where

$H_{k}=2 i\left[\begin{array}{cc}0 & -J_{1}-J_{2} e^{-i k} \\ J_{1}+J_{2} e^{i k} & 0\end{array}\right]$.

The above Hamiltonian can be diagonalized where the eigenvalues are given by

$$
\epsilon_{k}^{ \pm}= \pm 2 \sqrt{J_{1}^{2}+J_{2}^{2}+2 J_{1} J_{2} \cos k} .
$$

Clearly, the gap vanishes at $J_{1}= \pm J_{2}$ for $k=\pi$ and 0 respectively with the critical exponents $\nu$ and $z$ both being equal to unity. Feng, Zhang and Xiang [39] showed that this vanishing energy gap signals a topological phase transition between the two phases of the model at $J_{1}<$ $J_{2}$ and $J_{1}>J_{2}$. Interestingly, this model can be mapped to a one-dimensional transverse Ising model by a duality transformation [39, 40, 41].

In terms of a new set of basis functions given by

$\psi_{1 k}=\frac{1}{\sqrt{2}}\left(\begin{array}{c}1 \\ i\end{array}\right)$ and $\psi_{2 k}=\frac{1}{\sqrt{2}}\left(\begin{array}{c}1 \\ -i\end{array}\right)$,

the above Hamiltonian can be recast to the form

$\tilde{H}_{k}=2\left[\begin{array}{cc}\frac{J_{+}+J_{-}}{2}+\frac{J_{+}-J_{-}}{2} \cos k & -\frac{J_{+}-J_{-}}{2} \sin k \\ -\frac{J_{+}-J_{-}}{2} \sin k & -\frac{J_{+}+J_{-}}{2}-\frac{J_{+}-J_{-}}{2} \cos k\end{array}\right]$

where $J_{ \pm}=J_{1} \pm J_{2}$. We study the dynamics of the spin chain by varying the term $J_{-}$of Hamiltonian (9) using the quenching rule $J_{-}=-\left|\frac{t}{\tau}\right|$ where $t$ varies from $-\infty$ to $+\infty$. Here, the quantum critical point occurs at $t=0$ and it is at this point where the parameter $J_{-}$is reversed to bring it back to its initial value at the final time. It is to be noted that the off-diagonal terms in the Hamiltonian (9) becomes time-dependent in the present quenching scheme making the analytical solution difficult. However, the situation can be easily saved by making an appropriate unitary transformation as shown below: In the limit of large $t(t \rightarrow \pm \infty)$, the eigenvectors of the Hamiltonian are

$$
\begin{aligned}
\left|e_{1 k}\right\rangle & =\frac{1}{\sqrt{2(1+\sin (k / 2))}}\left[\cos \frac{k}{2}\left|\psi_{2 k}\right\rangle+\left(1+\sin \frac{k}{2}\right)\left|\psi_{1 k}\right\rangle\right] \\
\left|e_{2 k}\right\rangle & =\frac{1}{\sqrt{2(1+\sin (k / 2))}}\left[-\cos \frac{k}{2}\left|\psi_{1 k}\right\rangle+\left(1+\sin \frac{k}{2}\right)\left|\psi_{2 k}\right\rangle\right]
\end{aligned}
$$

where $\left|e_{1 k}\right\rangle$ is the ground state in the limit $t \rightarrow-\infty$. A unitary transformation generated by the matrix $U$ constructed from the above eigenvectors leads to the final the Hamiltonian suitable for the present form of quenching and is given by

$$
\begin{aligned}
H_{k}^{\prime} & =U^{\dagger} \tilde{H}_{k} U \\
& =2\left[\begin{array}{cc}
J_{-}(t) \sin \left(k^{\prime}\right) & J_{+} \cos \left(k^{\prime}\right) \\
J_{+} \cos \left(k^{\prime}\right) & -J_{-}(t) \sin \left(k^{\prime}\right)
\end{array}\right],
\end{aligned}
$$

where the time dependence is now entirely shifted to diagonal terms of the Hamiltonian. The quantum critical point is at $J_{-}=0$ for the mode $k^{\prime}=\pi / 2$. Also, the mode $k^{\prime}$ in Eq. (10) is half of mode $k$ in Eq (8). Henceforth, we will refer $k^{\prime}$ as $k$ and appropriately redefine the Brillouin zone. The presence of a single QCP precisely at $t=0$ renders the analytical calculation easier and so we chose Kitaev model over other exactly solved models for the present study. We note that the results of this model can be extended to any other Jordan-Wigner solvable models.

\section{RESULTS}

In this section, we shall present the main results of this work. The $2 \times 2$ reduced Hamiltonian matrix given in Eq. (10) can be interpreted as a Landau Zener Hamiltonian [28, 29] where the diagonal elements are the two bare (diabatic) energy levels which approach each other and the off-diagonal element $\Delta_{k}$ is the minimum gap between the instantaneous levels of the Hamiltonian. At time $t=0$, the energy gap between the instantaneous energy levels vanish for the mode $k=\pi / 2$ signaling a 9) quantum phase transition mentioned above. We shall assume that the system is prepared in its initial ground state $\left|e_{1 k}\right\rangle$ at $t \rightarrow-\infty$. At any instant $t$ during the evolution, a general state vector $\left|\psi_{k}(t)\right\rangle$ can be expressed as $\left|\psi_{k}(t)\right\rangle=c_{1, k}(t)\left|e_{1 k}\right\rangle+c_{2, k}(t)\left|e_{2 k}\right\rangle$ where $c_{i, k}(t) \quad(\mathrm{i}=1,2)$ denote the time-dependent probability amplitude for the bare state $\left|e_{i k}\right\rangle$.

The Schrödinger equation describing the evolution of the system is

$$
\begin{aligned}
i \frac{\partial}{\partial t} c_{1, k}(t) & =2 J_{-} \sin (k) c_{1, k}(t)+2 \cos (k) c_{2, k}(t) \\
i \frac{\partial}{\partial t} c_{2, k}(t) & =-2 J_{-} \sin (k) c_{2, k}(t)+2 \cos (k) c_{1, k}(t),
\end{aligned}
$$


with initial conditions $c_{1, k}(-\infty)=1, c_{2, k}(-\infty)=0$ and we have set $J_{+}=1$. The nonadiabatic transition probability in the final state is given by $\left|c_{2, k}(+\infty)\right|^{2}$. The above Schrödinger equations are solved exactly and the probability of excitation for the $k$-th mode, $p_{k}$, is

$$
\begin{aligned}
p_{k}(t \rightarrow \infty)= & \frac{1}{4}\left(1-e^{-2 \pi \alpha}\right) \\
& \times \quad\left|\frac{\Gamma(1-i \alpha / 2)}{\Gamma(1+i \alpha / 2)}+i \frac{\Gamma(1 / 2-i \alpha / 2)}{\Gamma(1 / 2+i \alpha / 2)}\right|^{2},
\end{aligned}
$$

where $\alpha=\tau \cos ^{2}(k) / \sin (k)$. The density of defects can be obtained by integrating the probability of excitations $p_{k}$ over the Brillouin zone and is given by

$n=\frac{1}{2 \pi} \int_{-\pi}^{\pi} p_{k}(t \rightarrow \infty) d k=\frac{1}{\pi} \int_{0}^{\pi} p_{k}(t \rightarrow \infty) d k$.

The parameter $\alpha$ measures the effective rate of driving. It is $\alpha$, not $\tau$ which determines the diabatic $(\alpha \rightarrow 0)$ and adiabatic $(\alpha \rightarrow \infty)$ limit 8,42 . The gap varies with wave vector $k$, so does $\alpha$ and for the modes close to the critical mode $(k=\pi / 2), \alpha \sim k^{2} \tau$. We defer the calculational details to the Appendix.

Let us first analyze the exact expression given in Eq. (12) in different limits and compare it with $p_{k}$ obtained by direct numerical integration of the Schrödinger equation (11). It should be noted that the first expression in the modulus squared term of Eq. (12) is

$$
\frac{\Gamma(1-i \alpha / 2)}{\Gamma(1+i \alpha / 2)}=\frac{\Gamma(z)}{\Gamma(\bar{z})}=\frac{\Gamma(z)}{\overline{\Gamma(z)}}
$$

which is a unit vector with argument $-2 \theta_{1}$ where $\theta_{1}$ is the argument of $\Gamma(1+i \alpha / 2)$. Similarly the second expression in the modulus squared term is also a unit vector with angle $-2 \theta_{2}$ and hence modulus squared term in Eq. (12) reduces to

$2+2 \sin \left(2 \theta_{2}-2 \theta_{1}\right)$

Therefore, the final expression for the probability of excitations is

$p_{k}(t \rightarrow \infty)=\frac{1}{4}\left(1-e^{-2 \pi \alpha}\right) \times\left|2+2 \sin \left(2 \theta_{2}-2 \theta_{1}\right)\right|$

Using the properties of $\Gamma$ function [43], we have

$$
\begin{gathered}
\theta_{1}=\frac{\alpha}{2} \psi(1)+\left(\sum_{l=0}^{\infty} \frac{\alpha}{2(1+l)}-\tan ^{-1}\left(\frac{\alpha}{2(1+l)}\right)\right) \\
\left.\theta_{2}=\frac{\alpha}{2} \psi\left(\frac{1}{2}\right)+\left(\sum_{l=0}^{\infty} \frac{\alpha}{2(1 / 2+l)}-\tan ^{-1}\left(\frac{\alpha}{2(1 / 2+l)}\right)\right) 16\right)
\end{gathered}
$$

where $\psi(z)$ is the well known Digamma function [43]. The analytical expression for $p_{k}$ is obtained by substituting $\theta_{1}$ and $\theta_{2}$ in $\mathrm{Eq}(15)$. The result obtained by numerical integration of the Schrödinger equation is presented in Fig. (1) where we also plot the analytical expression after substituting numerically obtained values of $\theta_{1}$ and $\theta_{2}$ in Eq. (15).

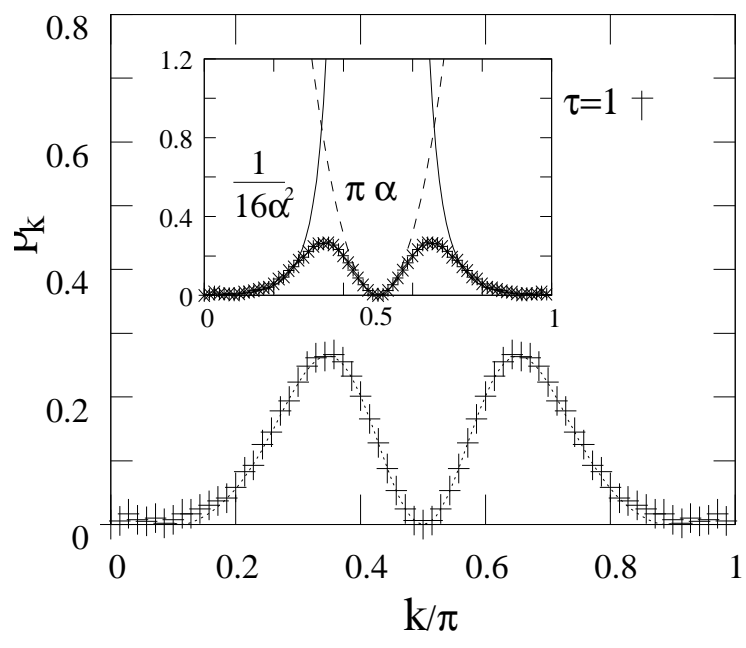

FIG. 1: Variation of $p_{k}$ vs $\mathrm{k}$ for $\tau=1$. The data with ' + ' sign represent the numerical solution whereas the dashed line corresponds to the analytical expression given in Eq. 12 As explained in the text, the region near $k=\pi / 2$ varies linearly with $\alpha=\tau \cos ^{2} k / \sin k$ whereas that away from $k=\pi / 2$ as $1 / \alpha^{2}$. The inset also shows the intersection of the two limits at a particular mode $k_{0}$. The dotted line in the inset goes as $\pi \alpha$ and the thin line falls as $1 / 16 \alpha^{2}$.

The behavior of $p_{k}$ as a function of the wave vector $k$ can be explained by making resort to the Landau-Zener interpretation discussed before. For the modes close to $k=0$ (which are away from the critical mode $k=\pi / 2$ ), the minimum gap $\Delta_{k}$ is relatively large, or more precisely $\Delta_{k}^{2} \tau>>1$. Hence, these modes evolve adiabatically remaining close to the instantaneous ground state throughout the quenching. For the critical mode $k=\pi / 2$, gap is zero or in other words the relaxation time (inverse of the gap) is diverging which results to the complete freezing of dynamics. The system stays in its initial ground state throughout the evolution which also happens to be the ground state at $t \rightarrow+\infty$ for the present scheme of quenching. We therefore encounter a situation where the mode for which instantaneous energy gap is zero has simultaneously zero excitation probability which is in contrast to the forward case where the probability of excitations is unity for the critical mode. Similarly, for the modes near $k=\pi / 2$ where the gap is still very small, the system stays closer to the initial state due to large relaxation time leading to a final state similar to the ground state. We therefore conclude that the transition probability vanishes in either limits $k \rightarrow 0$ and $k \rightarrow \pi / 2$ and a peak is expected at a wave vector $k_{o}$ lying somewhere in the middle as shown in Fig. (1).

The instantaneous excitation at an instant $t$ is defined as the probability of finding the system in the instantaneous excited eigenstate of the Hamiltonian (10). The variation of the instantaneous excitation probability as a function of time presented in Fig.(2) reflects the explanation presented above.

In the limit of small $\alpha$ (i.e., either $\tau$ small or $k \rightarrow \pi / 2$ ), 


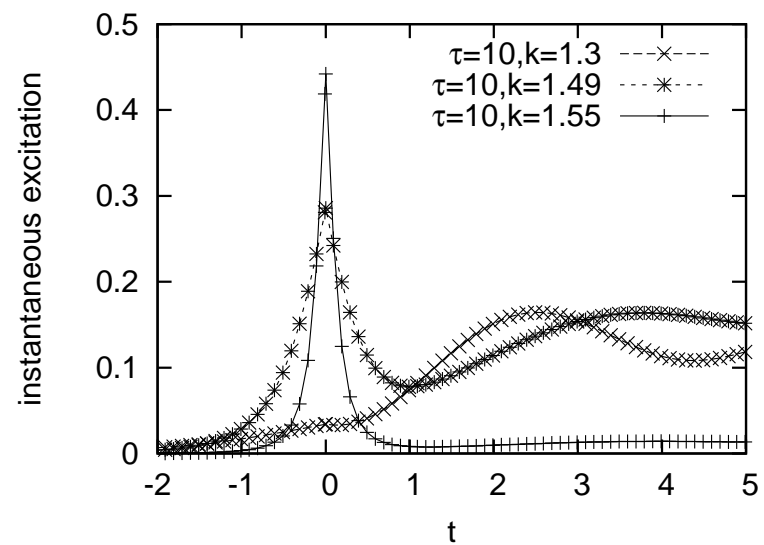

FIG. 2: Instantaneous excitation probabilities vs time for three different modes. The mode $k=1.49$ is closer to the $\pi / 2$ mode and shows a diabatic behavior i.e., the small gap and large relaxation time makes the system unable to change appreciably from the initial state and therefore the instantaneous excitation decreases in magnitude for $t>0$ when it is retracing its path. On the other hand, the system tries to follow the instantaneous ground state for $k=1.3$ which is in the adiabatic limit and the excitation keeps on increasing till the effect of finite gap persists. Finally, for the mode $k=1.55$ which is closest to the critical mode $k=\pi / 2$, decrease in instantaneous excitation for $t>0$ is prominently shown.

we get a simplified expression

$2 \theta_{2}-2 \theta_{1}=-2 \alpha \ln 2$.

Substituting Eq. (17) in Eq (15), we get the expression for $p_{k}$ in the small $\alpha$ limit as

$p_{k}=\frac{1}{4}\left(1-e^{-2 \pi \alpha}\right) \times(2-2 \sin (2 \alpha \ln 2))$.

which correctly predicts the curve for small $\alpha$ along with the peak of the curve.

It would be useful to calculate $p_{k}$ in the two extreme limits, namely, $\alpha \rightarrow 0$ and $\alpha \rightarrow \infty$.

The $\alpha \rightarrow 0$ limit can be obtained directly from Eq. ( 12) whereas the large $\alpha$ limit is obtained from using the asymptotic expansion of the Gamma function in Eq (12). Thus, we have

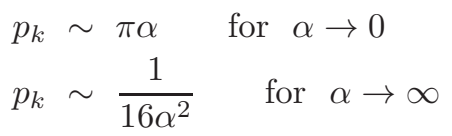

which is also shown in the inset of Fig. 1. As discussed already, the $\alpha \rightarrow 0$ behavior is expected near the critical mode $k=\pi / 2$ whereas large $\alpha$ behavior can be observed for the modes $k$ away from $\pi / 2$. It is also interesting to note that the exact solution we present here reduces to the adiabatic transition probability scaling $1 / \alpha^{2}$ in the limit of large $\alpha$ as expected from the quantum mechanical adiabatic theorem [29, 44]. This feature is more transparent in Fig. 3 where the variation of $p_{k}$ with $\tau$ for two different values of $k$, one near $k=\pi / 2$ and the

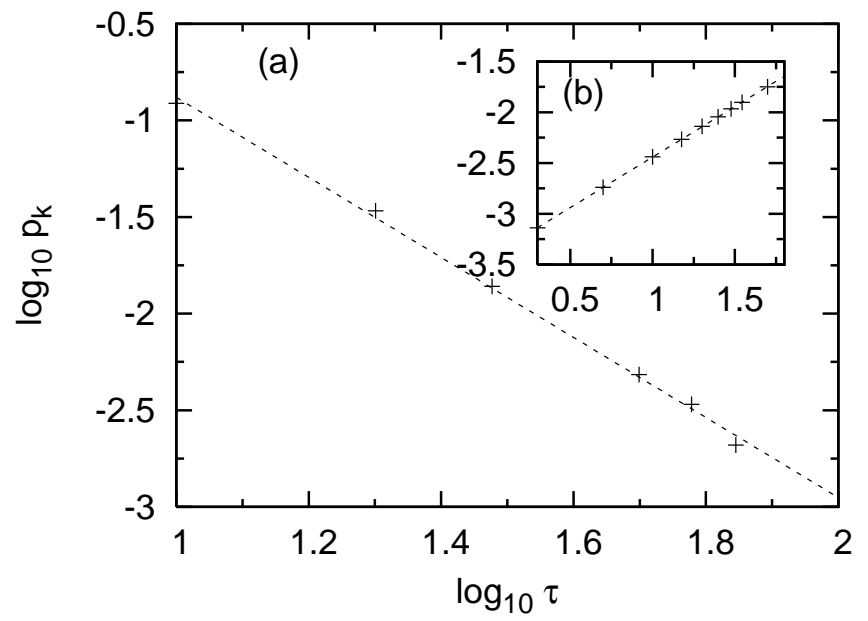

FIG. 3: Variation of $\log p_{k}$ vs $\log \tau$ for two different $\mathrm{k}$ values. Fig (a) corresponds to $\mathrm{k}=1.3$ where $1 / \tau^{2}$ behavior is expected. The dots are the numerically obtained values where as the fitted line has a slope -2. Fig (b) (inset), on the other hand is for $k=1.56$ where $p_{k}$ increase linearly with $\tau$. Once again a log-log plot shows a slope of 1 as expected from theory.

other away from $k=\pi / 2$, is shown depicting the linear increase with $\tau$ and decrease as $1 / \tau^{2}$ respectively.

The mode $k_{0}$ at which the peak in $p_{k}$ occurs is approximately given by the point of intersection of two limiting behaviors given in Eq. 15 and is given by

$$
\frac{\cos ^{2}\left(k_{0}\right)}{\sin \left(k_{0}\right)}=\left(\frac{1}{16 \pi}\right)^{1 / 3} \frac{1}{\tau}
$$

The value of $\alpha$ at $k_{0}$ is given by

$$
\alpha\left(k=k_{0}\right)=\sqrt[3]{1 / 16 \pi}
$$

which is independent of $\tau$. This implies that the maximum value of $p_{k}$ is independent of $\tau$ as shown in Fig 4 A rough estimate of this value can be obtained from Eq (18) after substituting $\alpha$ at $k_{0}$ and is found to be close to 0.25 .

Let us shift our attention to estimating the density of defects $n$ in the final state at $t \rightarrow \infty$. The variation of density of defects with the rate of quenching $\tau$ is obtained by integrating the probability of excitations over the Brillouin zone given by Eq. (13). We find that $n(\tau)$ as a function of $\tau$ shows a peak at a particular quenching rate $\tau_{0}$ and eventually follows a $1 / \sqrt{\tau}$ decay for very large $\tau$. The $1 / \sqrt{\tau}$ behavior is justified by noting the fact that for very large $\tau, k_{0}$ shifts towards $\pi / 2$ where $\cos ^{2}(k) / \sin (k) \sim k^{2}$. In this large $\tau$-limit therefore, $p_{k}$ scales as $p_{k} \sim p_{k}\left(\tau k^{2}\right)$ resulting to the Kibble-Zurek scaling of the defect density given as $n \sim 1 / \sqrt{\tau}$. Fig. 5 shows the variation of density of defects with $\tau$ in the large $\tau$ limit with a $1 / \sqrt{\tau}$ behavior whereas the inset of Fig. 5 corresponds to the $n$ vs $\tau$ behavior for the entire range of $\tau$ depicting the peak as described above.

An interesting observation is that for the present quenching scheme, the density of defects in the final state 


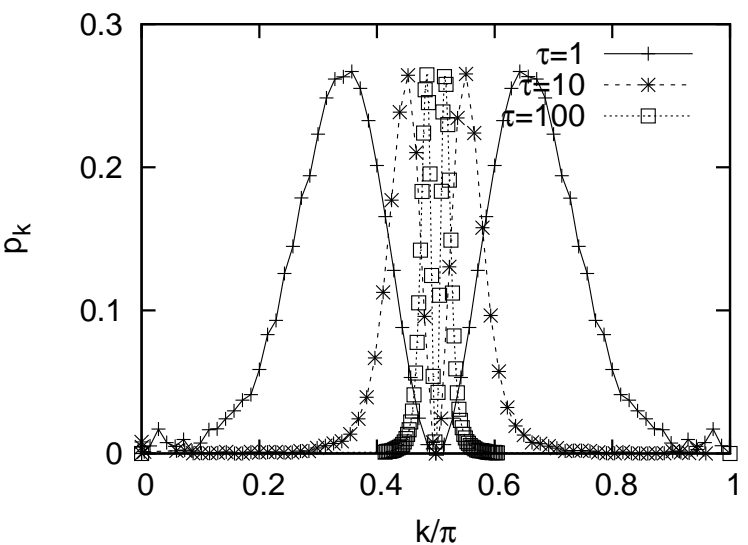

FIG. 4: The variation of $p_{k}$ for different values of $\tau$ showing that the maximum value of $p_{k}$ is independent of $\tau$

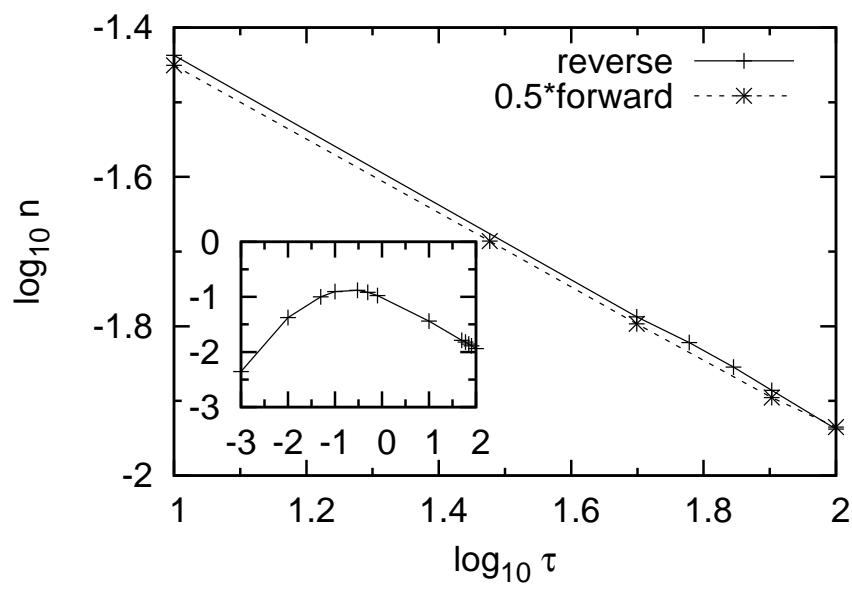

FIG. 5: The main part of the figure shows the variation of density of defects $n$ for relatively higher values $\tau$ for reverse quenching. Also plotted is half times the density of defects produced while forward quenching and it is clear that reverse quenching is close to half of the linear quenching. Inset shows $n$ vs. $\tau$ for a wider range of $\tau$ where a peak is observed for a relatively smaller value of $\tau$

is close to half of that in the forward quenching i.e., the case where $J_{-}$is linearly quenched from $-\infty$ to $\infty$, see Fig (5). This is because the maximum value of $p_{k}$ in the reverse case is one-fourth that of the forward case making the area under one of the peaks to be close to one fourth that of the linear.

It is also illustrative to compare the non-adiabatic transition probability $p_{k}(t \rightarrow+\infty)$ as a function of $k$ for reverse quenching, forward quenching as well as half quenching i.e., $p_{k}(t=0)$. Fig. 6] a) suggests that the density of defects (area under the $p_{k}$ vs $k$ curve) does not change appreciably in reverse quenching as compared to the half quenching. However, there is a reorganization of $p_{k}$ in the wave-vector space keeping the density of defect nearly constant. To justify the above statement, we
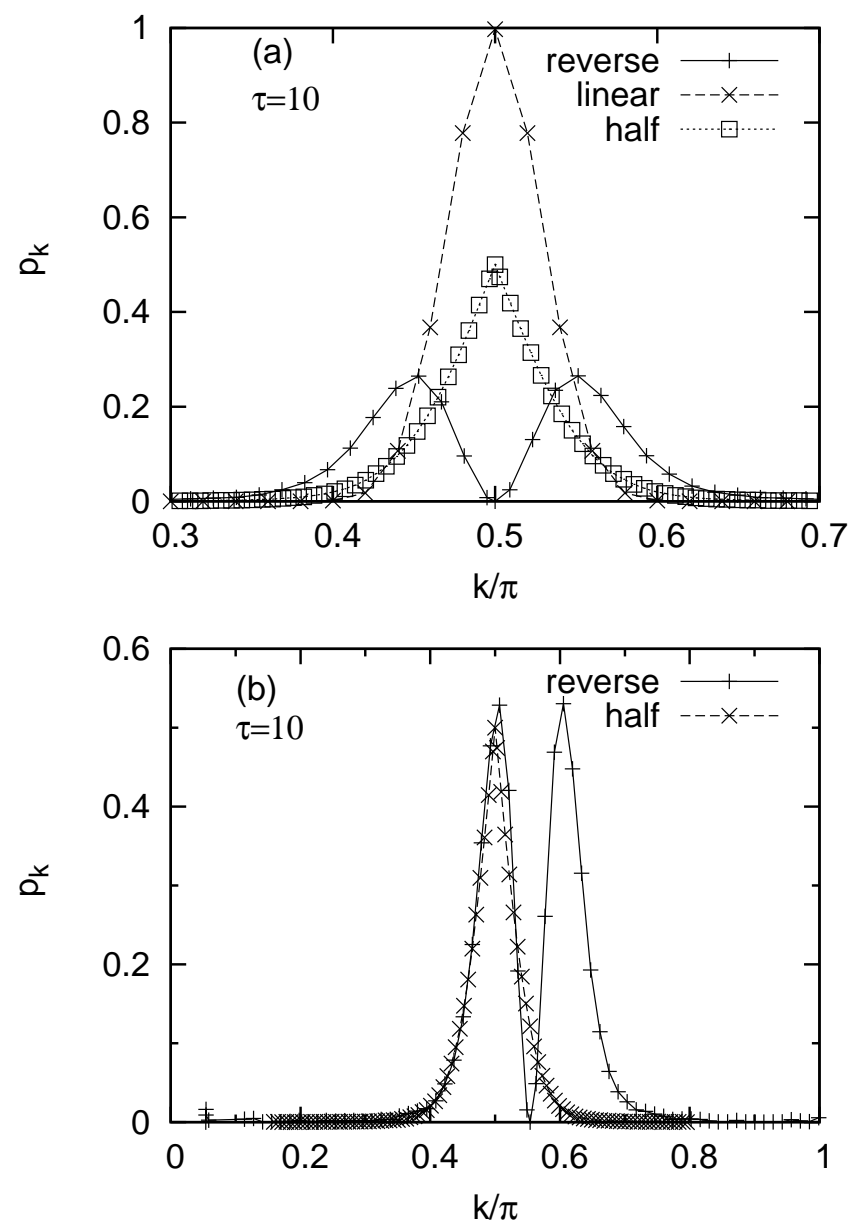

FIG. 6: a) A comparison between probability of excitations for reverse quenching, forward quenching and $p_{k}(t=0)$. is presented The range of $k$ is appropriately chosen for the clarity of presentation. Fig. 6b)shows that the defect generated in the reverse case, which is the area under the $p_{k}$ vs. $k$ curve, is approximately equal to the defect generated at $t=0$. To highlight this, we have doubled $p_{k}$ for the reverse case and overlapped one of its peaks with $p_{k}(t=0)$ by appropriate shifting of the $x$-axis.

have doubled the peaks in the reverse case and appropriately shifted the $\mathrm{x}$-axis to match one of its peaks with $p_{k}(t \rightarrow 0)$ in Fig 6(b). The peak is found to match almost identically to $p_{k}(0)$. In the passing, we note from Fig. (6a), that for very large $\tau$ when only the modes close to the critical mode contributes to the defects, the density of defects in the forward case is double that of half quenching.

We now shift our focus to the local von-Neuman entropy density [9] defined by

$S=-\frac{1}{\pi} \int_{0}^{\pi}\left(p_{k} \ln p_{k}+\left(1-p_{k}\right) \ln \left(1-p_{k}\right)\right) d k$

generated in the reverse quenching process. The entropy density is small for small as well as large values of $\tau$ 


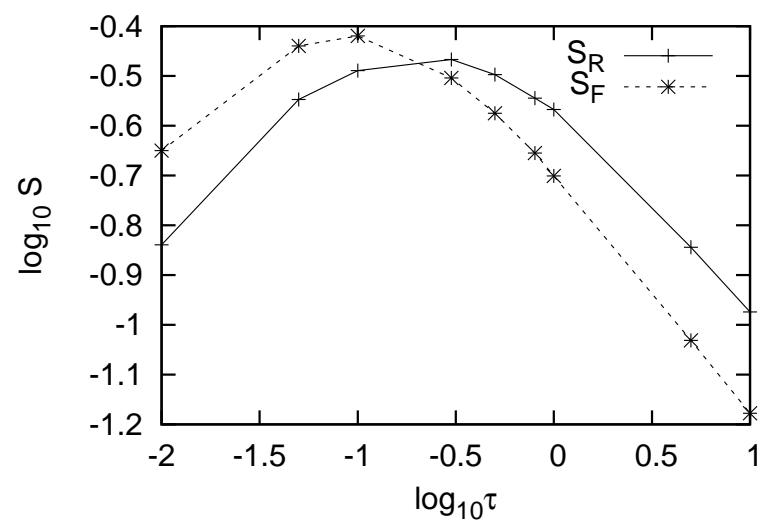

FIG. 7: Comparison of entropy of reverse and forward quenching.

and attains the maximum value at a characteristic timescale. A comparison between the entropy generated in the forward $\left(S_{F}\right)$ and the reverse case $\left(S_{R}\right)$ is shown in Fig. 7 Although qualitatively the curves are similar, an interesting difference is to be pointed out: $S_{R}$ is less than $S_{F}$ in the limit of small $\tau$ whereas $S_{R}$ exceeds $S_{F}$ for large $\tau$. This observation can be explained as follows: the integrand in Eq (20) is maximum when $p_{k}=0.5$. In reverse case, $p_{k}$ always remains less than 0.5 , i.e., it never reaches the maximally disordered state. For the forward case in the small $\tau$ (non-adiabatic) limit, $p_{k}$ is close to 0.5 in a larger region and hence entropy is large. In other words, the final state is more locally ordered following a reverse quenching than a forward quenching in the limit $\tau \rightarrow 0$. On the other hand, for the large $\tau$ limit in the forward case, $p_{k}$ increases sharply near the critical mode $k=\pi / 2$ and non-negligible only for wave vectors close to $\pi / 2$ resulting to relatively smaller entropy density.

In a recent work, Barankov and Polkovnikov [45] have proposed the concept of diagonal entropy given by

$S_{d}=-\sum_{n} \rho_{n n} \ln \rho_{n n}$

where $\rho_{n n}$ is the $n$-th diagonal element of the density matrix describing the system. One can interpolate it to obtain a time dependent diagonal entropy where $\rho_{n n}(t)$ is the diagonal elements of the density matrix in the instantaneous eigen basis. In our case, $\rho_{11}(t)=1-p_{k}(t)$ and $\rho_{22}(t)=p_{k}(t)$ where the excitations for each mode $k$ are calculated in the instantaneous eigen basis. Here, we compare the evolution of the diagonal entropy in the reverse and forward cases. We find that in the forward case the diagonal entropy increases monotonically with time and eventually saturates to the asymptotic value corresponding to the von-Neuman entropy whereas in the reverse case, a dip is observed immediately after the critical point.

The experimental realization of the Kitaev model has been proposed recently in systems of ultracold atoms and

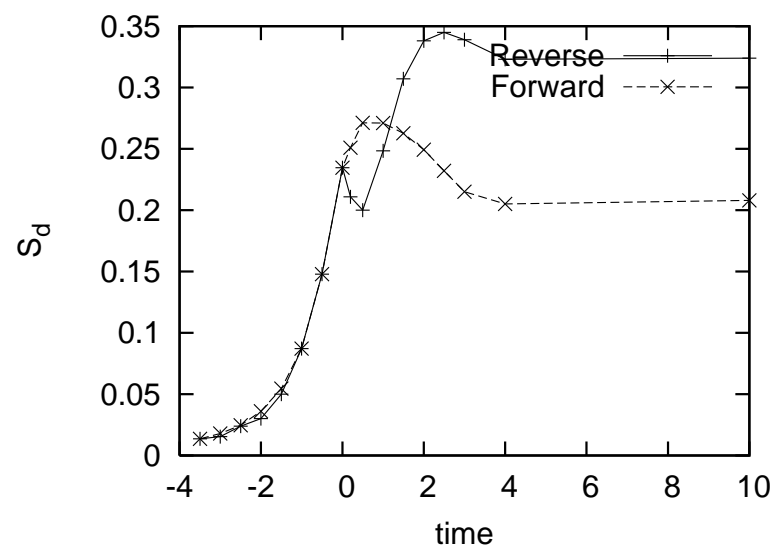

FIG. 8: Variation of diagonal entropy with time for $\tau=10$.

molecules trapped in optical lattices 31]. In this proposal, each of the couplings can be independently tuned using different microwave radiations. Once this is established, one can experimentally verify the reverse quenching case by looking at the defect density which actually corresponds to the number of bosons in the wrong spin state. It is also possible to investigate the spatial correlation function of the operator $i b_{n} a_{n+r}$ where $a_{n}$ and $b_{n}$ are Majorana fermions as defined before [16]. This spatial correlation function depends on $p_{k}$ which we have already obtained for the reverse case. Then the evolution of defect correlations can be detected by spatial noise correlation measurements as discussed in Ref. 46. Also, qualitative testing of reverse quenching can be done by varying a magnetic field in spin gap dimer compounds such as $\mathrm{BaCuSi}_{2} \mathrm{O}_{6}$ which undergo a singlet-triplet quantum phase transition at a critical field $B_{c}$. Our results suggest that the density of defects in the reverse case which correspond to residual singlets obtained by magnetization measurement would be close to half of the forward case.

\section{CONCLUSIONS}

In conclusion, we study the dynamics of a onedimensional Kitaev model using a reverse quenching scheme, by increasing the anisotropy parameter $J_{-}$linearly up to its quantum critical point at $t=0$ following which $J_{-}$is decreased at the same rate to bring it back to its initial value. We provide an exact solution of the Schrödinger equation and estimate the density of defects and the local entropy density in the final state. Comparison of the reverse quenching results to those of the corresponding forward quenching case leads to a few interesting observations. In the reverse case, the LandauZener transition probability $p_{k}$ vanishes for the critical mode $k=\pi / 2$ at which the instantaneous energy gap vanishes at $t=0$ whereas $p_{k}$ is maximum for the same mode in the forward case [16]. We show that $p_{k}$ increases 
linearly with $\alpha$ in small $\alpha$ (diabatic) limit whereas our result retrieves the expected $1 / \alpha^{2}$ fall of $p_{k}$ in the large $\alpha$ (adiabatic) limit as predicted from quantum mechanical adiabatic theorem; $\alpha$ is the effective rate parameter as defined in the text. Interestingly, the density of defects in the reverse case is close to half that of forward quenching. We have also compared the half quenching case with reverse quenching. A close inspection of $p_{k}$ as a function of $k$ as shown in Fig. 6 suggests that in the reverse case, $p_{k}$ gets reorganized in wave vector space with a peak at $k=k_{0} \neq \pi / 2$ keeping the density of defects approximately same as half quenching case. The value of this $k_{0}$ shifts to $\pi / 2$ for $\tau \rightarrow \infty$. We also show that the maximum value of the transition probability is independent of $\tau$. The local entropy density of the final state and time evolution of the diagonal entropy density are also explored. The possibility of experimental realization of the reverse quenching scheme using atoms trapped on optical lattices has also been pointed out.

\section{Acknowledgement}

AD acknowledges R. Moessner and the hospitality of MPIPKS, Dresden where the initial part of this work was done The authors acknowledge Diptiman Sen and Krishnendu Sengupta for critical comments. We also thank P. P. Kurur and Victor Mukherjee for many fruitful discussions.

\section{APPENDIX A: DETAILS OF EXACT CALCULATION}

We present here an outline of the calculational details leading to an exact solution for the reverse quenching case generalizing earlier studies [8, 28, 29] to the present case. Let us first consider a general Hamiltonian in the basis $|1\rangle$ and $|2\rangle$ as shown below

$H=\left[\begin{array}{cc}\epsilon_{1} & \Delta \\ \Delta^{*} & \epsilon_{2}\end{array}\right]$

where $\epsilon_{1}$ and $\epsilon_{2}$ are the two bare energy levels (diagonal elements) varying as $\sim t / 2 \tau$ and $-t / 2 \tau$ respectively (see Eq. (10) for comparison). If $|\psi(t)\rangle=c_{1}(t)|1\rangle+c_{2}(t)|2\rangle$, then one can write down the Schrödinger equation for $c_{1}(t)$ and $c_{2}(t)$. But before that we redefine $c_{1}(t)$ and $c_{2}(t)$ as follows

$c_{1}(t)=\tilde{c}_{1}(t) e^{-i \int_{-\infty}^{t} \epsilon_{1}^{\prime} d t^{\prime}}$

$c_{2}(t)=\tilde{c}_{2}(t) e^{-i \int_{-\infty}^{t} \epsilon_{2}^{\prime} d t^{\prime}}$.

The Schrödinger equation for $\tilde{c}_{2}$ is

$i \frac{\partial}{\partial t} \tilde{c}_{2}(t)=\Delta \tilde{c}_{1}(t) e^{-i \int_{-\infty}^{t}\left(\epsilon_{1}\left(t^{\prime}\right)-\epsilon_{2}\left(t^{\prime}\right)\right) d t^{\prime}}$.

One more transformation of the form

$\tilde{c}_{2}(t)=e^{-\frac{i}{2} \int_{-\infty}^{t}\left(\epsilon_{1}-\epsilon_{2}\right) d t^{\prime}} U_{2}(t)$ helps us to write the equation for $\tilde{c}_{2}$ in terms of $U_{2}$ in the following form

$\frac{\partial^{2}}{\partial t^{2}} U_{2}(t)+\left(\Delta^{2}-\frac{i}{2 \tau}+\frac{t^{2}}{4 \tau^{2}}\right) U_{2}(t)=0$

where we have substituted $\epsilon_{1}-\epsilon_{2}=t / \tau$. Now redefining a new variable

$$
z=\frac{t}{\sqrt{\tau}} e^{-i \pi / 4}
$$

one gets,

$\frac{\partial^{2}}{\partial z^{2}} U_{2}(z)+\left(m+\frac{1}{2}-\frac{z^{2}}{4}\right) U_{2}(z)=0$

where $m=i \Delta^{2} \tau$. By all these transformations, we are able to recast the Schrödinger equation in the form of Weber Differential equation [47] whose solutions are linear combination of well known Weber functions $D_{-m-1}(i z)$ and $D_{-m-1}(-i z)$ i.e.,

$U_{2}(z)=a D_{-m-1}(i z)+b D_{-m-1}(-i z)$

or going back to the notation of $\tilde{c}_{1}(t)$ and $\tilde{c}_{2}(t)$,

$$
\begin{aligned}
|\psi(t)\rangle & =\frac{i}{\Delta}\left[\partial_{t}-\frac{i t}{2 \tau}\right]\left[a D_{-m-1}(i z)+b D_{-m-1}(-i z)\right]|1\rangle \\
& +\left[a D_{-m-1}(i z)+b D_{-m-1}(-i z)\right]|2\rangle .
\end{aligned}
$$

But the initial condition demands that at $t \rightarrow-\infty$, $|\psi(t)\rangle \sim|1\rangle$ forcing $U_{2}(z)$ to be a function of only $D_{-m-1}(-i z)$ as $D_{-m-1}(-i z)$ goes to zero at $t \rightarrow-\infty$ but $D_{-m-1}(i z)$ does not as can be seen from the following asymptotic form of Weber functions

$$
\begin{array}{r}
D_{n}(z) \sim e^{-\frac{1}{4} z^{2}} z^{n}-\frac{\sqrt{2 \pi}}{\Gamma(-n)} e^{n \pi i r} e^{\frac{1}{4} z^{2}} z^{-n-1} \\
\text { for } \frac{\pi r}{4}<\arg (z)<\frac{5 \pi r}{4}
\end{array}
$$

where $r$ is either 1 or -1 , and

$D_{n}(z) \sim e^{-\frac{1}{4} z^{2}} z^{n}$ for $|\arg (z)|<\frac{3 \pi}{4}$

Therefore, $a=0$ and the form of $b$ can be obtained by these asymptotic forms along with the initial condition, which gives $b=\Delta \sqrt{\tau} e^{-\frac{\pi}{4} \Delta^{2} \tau}$.

Hence, Eq (A7), after using the derivative of Weber function, is

$$
\begin{aligned}
& |\psi \quad(t \leq 0)\rangle=e^{-\frac{\pi}{4} \Delta^{2} \tau} e^{i \frac{3 \pi}{4}}\left[(m+1) D_{-m-2}(-i z)\right. \\
& \left.-\quad i z D_{-m-1}(-i z)\right]|1\rangle+\Delta \sqrt{\tau} e^{-\frac{\pi}{4} \Delta^{2} \tau} D_{-m-1}(-i z)|2\rangle
\end{aligned}
$$

At $t=0$, the wavefunction is

$$
\begin{aligned}
|\psi(t=0)\rangle & =e^{-\frac{\pi}{4} \Delta^{2} \tau} e^{i \frac{3 \pi}{4}} \frac{\sqrt{\pi} 2^{-m / 2}}{\Gamma(1 / 2+m / 2)}|1\rangle \\
& +\Delta \sqrt{\tau} e^{-\frac{\pi}{4} \Delta^{2} \tau} \sqrt{\frac{\pi}{2}} \frac{2^{-m / 2}}{\Gamma(1+m / 2)}|2\rangle
\end{aligned}
$$


which is obtained by using the following property

$$
\lim _{s \rightarrow 0} D_{m}(s)=2^{m / 2} \frac{\sqrt{\pi}}{\Gamma(1 / 2-m / 2)}+O(s) .
$$

The wave function at $t>0$, which has the effect of reversing, should match with the wave function for $t<0$ at $t=0$. In the reverse case, the parameters $m$ and $z$ for $t>0$ are redefined as

$m^{\prime}=-i \Delta^{2} \tau$ and $z^{\prime}=\frac{-i t}{\sqrt{\tau}} e^{-i \pi / 4}$.

With these redefined $k^{\prime}$ and $z^{\prime}$, starting from Eq (A7), the wavefunction for $t<0$ are matched with that of $t>0$ at $t=0$ to obtain coefficients $a$ and $b$

$a=\frac{1}{2} \Delta \sqrt{\tau} e^{-\frac{\pi}{4} \Delta^{2} \tau} 2^{-m} \times\left[\frac{\Gamma(1-m / 2)}{\Gamma(1+m / 2)}-i \frac{\Gamma(1 / 2-m / 2)}{\Gamma 1 / 2+m / 2}\right]$

$$
b=\frac{1}{2} \Delta \sqrt{\tau} e^{-\frac{\pi}{4} \Delta^{2} \tau} 2^{-m} \times\left[\frac{\Gamma(1-m / 2)}{\Gamma(1+m / 2)}+i \frac{\Gamma(1 / 2-m / 2)}{\Gamma 1 / 2+m / 2}\right]
$$

We know that at $t \rightarrow \infty$, the excited state is $|2\rangle$ and hence the coefficient $\left|c_{2}\right|^{2}$ defines the excitation probability and is equal to

$c_{2}(t \rightarrow \infty) \sim \lim _{z \rightarrow \infty} a D_{-m^{\prime}-1}\left(i z^{\prime}\right)+b D_{-m^{\prime}-1}\left(-i z^{\prime}\right)(\mathrm{A} 1$

Once again, using the expression for $b$ and asymptotic expansion of Weber function in the definition of $\mid c_{2}(t \rightarrow$ $\infty)\left.\right|^{2}$ with properly identifying $\tau$ and $\Delta$ for a Kitaev model, we get Eq (12).
[1] S. Sachdev, Quantum Phase Transitions (Cambridge University Press, Cambridge, 1999).

[2] B. K. Chakrabarti, A. Dutta, and P. Sen, Quantum Ising Phases and Transitions in Transverse Ising Models, m41 (Springer-Verlag, Berlin, 1996).

[3] T. Kadowaki and H. Nishimori, Phys. Rev. E 58, 5355 (1998); P. Calabrese and J. Cardy, J. Stat. Mech: Theory Expt P04010 (2005), and Phys. Rev. Lett. 96, 136801 (2006); R. Schützhold, M. Uhlmann, Y. Xu, and U. R. Fischer, Phys. Rev. Lett. 97, 200601 (2006); C. Kollath, A. M. Läuchli, and E. Altman, Phys. Rev. Lett. 98, 180601 (2007); S. R. Manmana, S. Wessel, R. M. Noack, and A. Muramatsu, Phys. Rev. Lett. 98, 210405 (2007); M. Eckstein and M. Kollar, Phys. Rev. Lett. 100, 120404 (2008).

[4] K. Sengupta, S. Powell, and S. Sachdev, Phys. Rev. A 69, 053616 (2004); A. Das, K. Sengupta, D. Sen, and B. K. Chakrabarti, Phys. Rev. B 74, 144423 (2006).

[5] W. H. Zurek, U. Dorner, and P. Zoller, Phys. Rev. Lett. 95, 105701 (2005); B. Damski, Phys. Rev. Lett. 95, 035701 (2005).

[6] A. Polkovnikov, Phys. Rev. B 72, 161201(R) (2005).

[7] J. Dziarmaga, Phys. Rev. Lett. 95, 245701 (2005).

[8] B. Damski and W. H. Zurek, Phys. Rev. A 73, 063405 (2006).

[9] R. W. Cherng and L. S. Levitov, Phys. Rev. A 73, 043614 (2006).

[10] J. Dziarmaga, Phys. Rev. B 74, 064416 (2006); T. Caneva, R. Fazio, and G. E. Santoro, Phys. Rev. B 76, 144427 (2007).

[11] F. M. Cucchietti, B. Damski, J. Dziarmaga, and W. H. Zurek, Phys. Rev. A 75, 023603 (2007).

[12] V. Mukherjee, U. Divakaran, A. Dutta, and D. Sen, Phys. Rev. B 76, 174303 (2007).

[13] U. Divakaran and A. Dutta, J. Stat. Mech: Theory and Experiment P11001 (2007).

[14] A. Fubini, G. Falci and A. Osterloh, New J. Phys 9, 134 (2007).

[15] A. Polkovnikov and V. Gritsev, Nature Physics 4, 477 (2008)

[16] K. Sengupta, D. Sen, and S. Mondal, Phys. Rev. Lett.
100, 077204 (2008); S. Mondal, D. Sen, and K. Sengupta, Phys. Rev. B 78, 045101 (2008).

[17] D. Sen, K. Sengupta, and S. Mondal, Phys. Rev. Lett. 101, 016806 (2008); R. Barankov and A. Polkovnikov, Phys. Rev. Lett. 101, 076801 (2008); S. Mondal, K. Sengupta and D. Sen, arXiv:0808.1175 to appear in Phys. Rev. B (2008); C. De Grandi, R. A. Barankov, and A. Polkovnikov, Phys. Rev. Lett. 101, 230402 (2008)

[18] F. Pellegrini, S. Montangero, G. E. Santoro, and R. Fazio, Phys. Rev. B 77, 140404(R) (2008).

[19] D. Patane, A. Silva, L. Amico, R. Fazio, and G. Santoro, Phys. Rev. Lett, 101, 175701 (2008).

[20] V. Mukherjee, A. Dutta, and D. Sen, Phys. Rev. B 77, 214427 (2008).

[21] U. Divakaran, A. Dutta, and D. Sen, Phys. Rev. B 78, 144301 (2008).

[22] T. Caneva, R. Fazio, and G. E. Santoro, Phys. Rev. B 78, 104426 (2008).

[23] S. Deng, G. Ortiz and L. Viola, Europhys. Lett. 8467008 (2008).

[24] J. Dziarmaga, J. Meisner, and W. H. Zurek, Phys. Rev. Lett. 101, 115701 (2008).

[25] U. Divakaran, V. Mukherjee, A. Dutta, and D. Sen, arXiv:0807.3606, to appear in J. Stat. Mech: Theoery and Expt. (2009).

[26] V. Mukherjee and A. Dutta, to appear in J. Stat. Mech: Theory and Experiment (2009).

[27] A. Bermudez, D. Patane, L. Amico and M. A. MartinDelgado, arXiv:0811.3843 (2008).

[28] C. Zener, Proc. Roy. Soc. London Ser A 137, 696 (1932); L. D. Landau and E. M. Lifshitz, Quantum Mechanics: Non-relativistic Theory, 2nd ed. (Pergamon Press, Oxford, 1965).

[29] S. Suzuki and M. Okada, in Quantum Annealing and Related Optimization Methods, Ed. by A. Das and B. K. Chakrabarti (Springer-Verlag, Berlin, 2005), p. 185;

[30] I. Bloch, J. Dalibard, and W. Zwerger, Rev. Mod. Phys. 80, 885 (2008).

[31] L. -M. Duan, E. Demler and M. D. Lukin, Phys. Rev. Lett. 91, 090402 (2003); A. Micheli, G. K. Brennen and P. Zoller, Nature Physics 2, 341 (2006). 
[32] T. W. B. Kibble, J. Phys. A 9, 1387 (1976), Phys. Rep. 67, 183 (1980).

[33] W. H. Zurek, Nature (London) 317, 505 (1985), and Phys. Rep. 276, 177 (1996).

[34] L. E. Saddler, J. M. Higbie, S. R. Leslie, M. Vengalattore and D. M. Stamper-Kurn, Nature (London) 443, 312 (2006).

[35] D. A. Garanin and R. Schilling, Phys. Rev. B 66, 174438 (2002).

[36] A Kitaev, Ann. Phys. (N.Y.) 321, 2 (2006).

[37] E. Lieb, T. Schultz, and D. Mattis, Ann. Phys. (NY) 16, 407 (1961); E. Barouch and B. M. McCoy, Phys. Rev. A 3, 786 (1971); J. B. Kogut, Rev. Mod. Phys. 51, 659 (1979); J. E. Bunder and R. H. McKenzie, Phys. Rev. B 60, 344 (1999).

[38] H. D. Chen and Z. Nussinov, J. Phys. A 41, 075001 (2008); D. H. Lee, G.-M. Zhang, and T. Xiang, Phys. Rev. lett. 99, 196805 (2007).

[39] X. Y. Feng, G. M Zhang and T. Xiang, Phys. Rev. Lett 98, 087204 (2007).
[40] H. W. Capel and J. H. H. Perk, Physica A 87, 211 (1977); J. H. H. Perk, H. W. Capel and Th. J. Siskens, Physica A 89, 304 (1977).

[41] W. Brzezicki, J. Dziarmaga and A. M. Oles, Phys. Rev. B 75, 134415 (2007); Z. Nussinov and G. Ortiz, arXiv:0812.4309 (2008).

[42] N. V. Vitanov, Phys. Rev. A 59, 988 (1999).

[43] M. Abramowitz and I. A. Stegun Handbook of Mathematical functions Ed.9, (Courier Dover Publications,1965).

[44] A Messiah,Quantum Mechanics (Courier Dover Publications, 1999).

[45] Roman Barankov and Anatoli Polkovnokov, arXiv:0806.2862

[46] E. Altman, E. Demler and M. D. Lukin, Phys. Rev. A 70, 013603 (2004).

[47] E. T. Whittaker and G. N. Watson, A course of Modern Analysis (Cambridge University Press, Cambridge UK, 1958). 